\title{
Museu Virtual: Pantógrafos e Homotetia em aulas de Matemática
}

\section{Soraya Barcellos Izar}

Colégio de Aplicação-UERJ e Colégio Pedro II

soizar@gmail.com

\begin{abstract}
Resumo
O objetivo deste artigo é sugerir possibilidades de uso de atividades para uma aula de geometria direcionada a alunos do $7^{\circ}$ ano (11-13 anos) do ensino fundamental, integrando recursos dinâmicos com a observação orientada e mediada pelo professor. Foram utilizados os applets de pantógrafos virtuais do site do Museu Universitário de História Natural e da Instrumentação Científica da Universidade de Modena e Reggio Emilia. A Homotetia foi a transformação plana escolhida por estimular a visualização da proporcionalidade entre duas figuras posicionadas em um determinado local do plano e a compreensão dos elementos e características da transformação: forma e tamanho da figura transformada em relação à figura original.
\end{abstract}

Palavras-chave: Proposta de atividades. Pantógrafos. Aplicativos Dinâmicos. Transformações Pontuais. Ensino Fundamental

\section{Virtual Museum: Pantographs and Homotetia in Mathematics Classes}

\begin{abstract}
The objective of this article is to suggest possibilities of using activities for a geometry class directed to students of the 7th grade (11-13 years) of elementary school, integrating dynamic resources with guided observation and mediated by the teacher. We used the virtual pantograph applets from the Museo Universitario di Storia Naturale website and from the Strumentazione Scientifica Università degli studi di Modena i Reggio Emilia as a dynamic resource. Homotetia was the plane transformation chosen by stimulating the visualization of proportionality between two figures positioned in a given place of the plane and the understanding of the elements and characteristics of the transformation: shape and size of the transformed figure in relation to the original figure.
\end{abstract}

Keywords: Proposal of activities. Pantographs. Applets. Point Transformations. Elementary School. 


\section{Introdução}

Estimular a visualização da proporcionalidade existente entre duas grandezas gráficas pode ser um interessante exercício para a evolução do pensamento geométrico. Com esse objetivo Izar e Bairral (2016) elaboraram uma série de atividades direcionadas aos alunos do $6^{\circ}$ e do $7^{\circ}$ anos do ensino fundamental que contribuísse para tal visualização. Dentre elas, o bloco de atividade dos Pantógrafos Virtuais foi planejado para que os alunos do $7^{\circ}$ ano do ensino fundamental pudessem reconhecer as características e propriedades de figuras homotéticas ampliadas ou reduzidas em função de uma razão. Os applets ${ }^{1}$ de pantógrafos virtuais do site $^{2}$ do Museo Universitario di Storia Naturale e dela Strumentazione Scientifica Università degli studi di Modena e Reggio Emilia foram utilizados como recurso para mediar as observações dos alunos.

Visualizar para Ferreira (1986, p.1784) é "formar ou conceber uma imagem mental de algo (que não se tem ante os olhos no momento)". Veloso (1998) relaciona o termo visualização à construção e à manipulação de imagens mentais. Em sintonia com Veloso, Bairral (2009, p. 61) destaca que a "visualização é um processo que vai além da mera observação de algo. Além de observar um objeto o indivíduo faz associações. Ainda que seja aparentemente automática e individualizada esta é uma importante atividade cognitiva."

Ambientes virtuais que disponibilizam aplicativos capazes de mover, rotacionar, deformar, vincular e relacionar elementos podem se tornar ferramentas relevantes para auxiliar na visualização ao longo do processo de ensino e de aprendizagem de Geometria. Dentre os variados recursos, os applets surgem como opções interessantemente dinâmicas. Bairral (2009) destaca que utilização do applet pode ser um recurso interessante quando direcionado a estimular e potencializar a visualização dos alunos. Oliveira (2010) ressalta que os instrumentos tecnológicos servem para mediar ações concretas das pessoas sobre o mundo e o mundo. As imagens utilizadas no desenvolvimento das atividades intencionaram mediar experiências para que o conceito de homotetia fosse desenvolvido pelos alunos.

O bloco de atividades dos pantógrafos tem como objetivo proporcionar ao aluno uma experiência dinâmica, que possa contribuir para a visualização dos elementos e caraterísticas da homotetia $^{3}$ (Figura 1), a semelhança e a posição das figuras, assim como promover a reflexão sobre

\footnotetext{
1 “Applets são pequenos programas que são executados dentro de páginas da web. São escritos em linguagem Java e de livre acesso aos interessados" (BAIRRAL, 2007, p. 49).

${ }^{2}$ Disponível em: http://archivioweb.unimore.it/theatrum/macchine/_00lab.htm. Acesso em: 28 nov. 2016.

3 "Homotetia é a transformação que associa dois a dois os pontos do plano, de tal modo que pares de pontos correspondentes estão alinhados com um ponto fixo e a razão das distâncias deste ponto fixo aos pares de pontos correspondentes é constante" (RODRIGUES, 1997, p. 86).
} 
as propriedades geométricas estruturais do Pantógrafo de Scheiner em um ambiente em que os alunos, nativos digitais, se identificam e trabalham com desenvoltura.

Figura 1 - Polígonos homotéticos em relação ao ponto Q e de razão igual a 1⁄2.

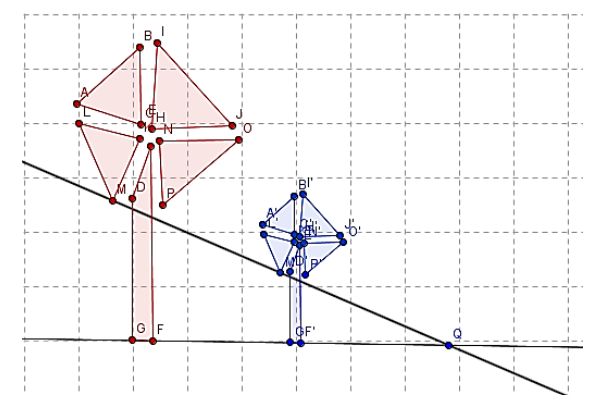

Fonte: Elaborada pela autora no GeoGebra.

\section{Sobre os pantógrafos}

Antes da computação gráfica, a representação gráfica de mapas não era tarefa simples. A complexidade das linhas de contorno das regiões representadas em escalas diferentes necessitava de um instrumento que respeitasse a sinuosidade das linhas mantendo o escala do mapa. O pantógrafo de Scheiner, também conhecido como pantógrafo de homotetia, atendia a essa necessidade (Figura 2).

Figura 2 - Pantógrafo de madeira e virtual (Scheiner) para ampliação e reducão.
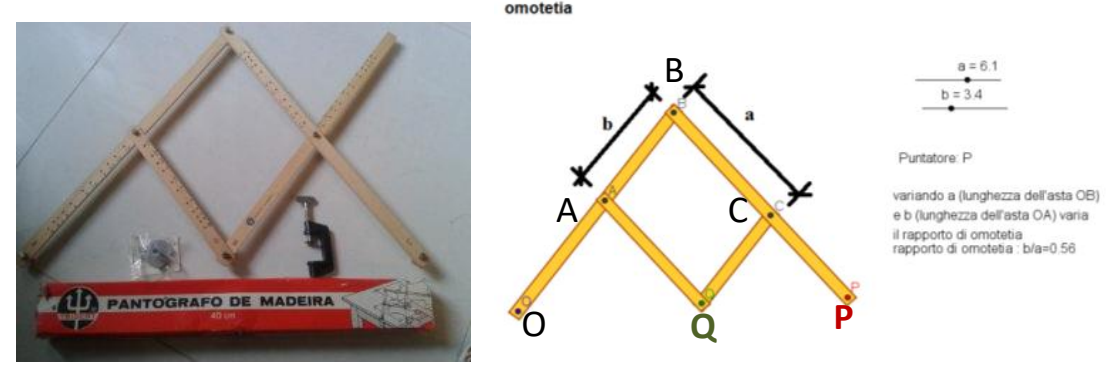

Fontes: http://img.olx.com.br/images/68/688529102427803.jpg http://www.macchinematematiche.org/images/macchine/trasformazioni/simulazioni/omotetia .html.

O mesmo principio de funcionamento do pantógrafo é utilizado em pantógrafos na engenharia mecânica. Denominados de fresadora pantográfica, fresadora pantógrafo ou fresa gravadora, reproduzem gravações para estampos de moedas, medalhas, placas com textos entre outras aplicações. As portas corrediças pantográficas que fecham a cabine de antigos elevadores, exemplificam outra utilização do conceito.

O pantógrafo de Scheiner é um instrumento que amplia ou reduz proporcionalmente desenhos, a partir de réguas que se articulam formando paralelogramos. Os princípios matemáticos, que regem o funcionamento dos pantógrafos partem da teoria de Descartes sobre os paralelogramos, 
inspiraram o padre jesuíta alemão Christofer Scheiner ${ }^{4}$, em 1603, a desenvolver o instrumento para utilização em diferentes âmbitos.

Os applets da seção 3 da mostra "Theatrum Machinarum"(strumenti per la geometria) ${ }^{5}$ foram escolhidos por estarem relacionados ao conteúdo planejado, além do formato da apresentação e do caráter interdisciplinar e dinâmico da exposição das máquinas (Figura 3).

\section{Figura 3 - Print da tela do website do Museo Universitario di Storia Naturale e Print da tela do website da Associazione Macchine Matematiche}
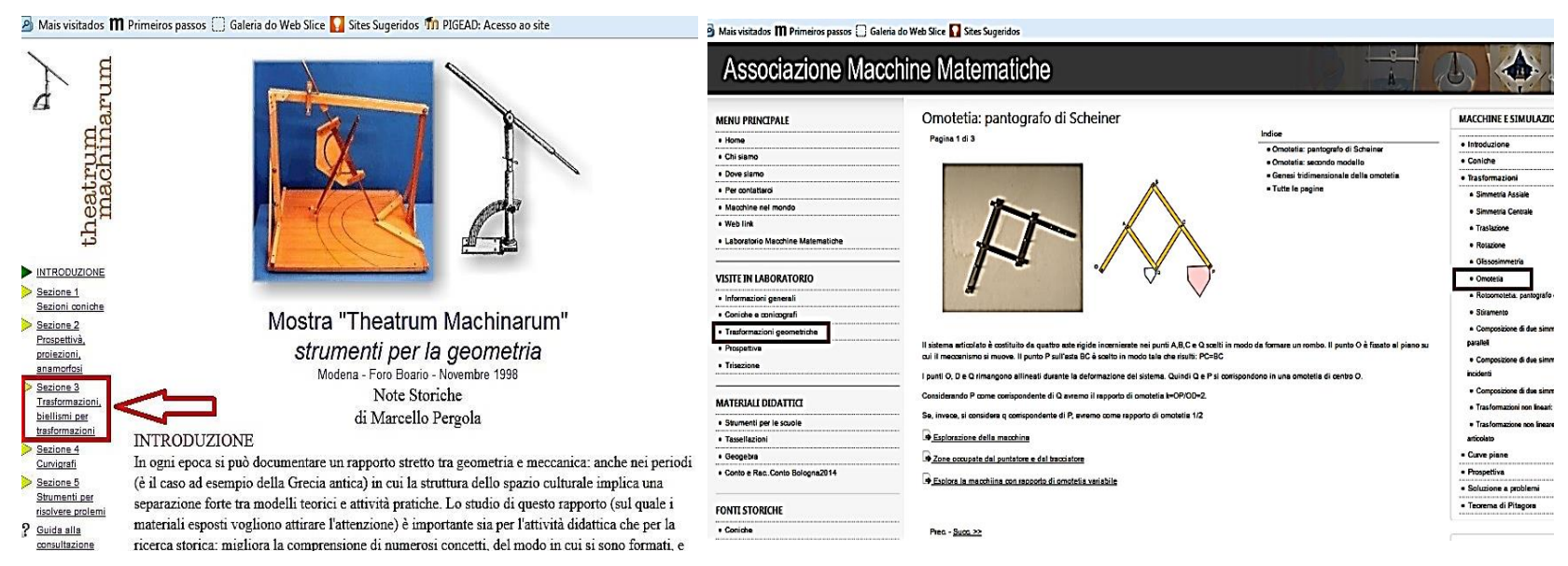

Fonte: Editada pela autora.

O kit "TRASFORMAZIONI"," composto por pantógrafos de translação, simetria axial e simetria central, rotação, homotetia e estiramento, e as perguntas do "Livello Base" disponibilizado pelo site Associazone Macchine Matematiche (Figura 3) inspiraram a elaboração das questões da folha de atividades, relativas à analise e à construção das estruturas dos pantógrafos.

\section{O Bloco de Atividades dos Pantógrafos}

O bloco de atividades dos Pantógrafos foi planejado para abordar o conteúdo sobre transformações isométricas (simetria central, simetria axial, translação, rotação) e as transformações não isométricas (homotetia), com os alunos do $7^{\circ}$ ano, destacando as relações métricas e de posição entre a figura original e a figura transformada.

Ao estimular a execução de atividades com os pantógrafos virtuais os alunos podem visualizar que a homotetia vai além da semelhança entre as figuras, pois a figura homotética fica

\footnotetext{
${ }^{4}$ Disponível em:

http://www.macchinematematiche.org/cataoghi/occhioemano/catalogoweb/approfondimenti/SCHEINER.pdf. Acesso em: 28 nov. 2016

${ }^{5}$ Disponível em http://www.macchinematematiche.org/cataoghi/theatrum/macchine/_00the.htm. Acesso em 28 nov. 2016.

${ }^{6}$ Disponível em http://www.macchinematematiche.org/images/kit/kit\%20nuovi.pdf. Acesso em 28 nov.2016.

${ }^{7}$ Disponível em http://www.macchinematematiche.org/images/visite_laboratorio/Omotetia_Base.pdf. Acesso em 28 nov. 2016
} 
restrita a uma determinada posição no plano devido ao posicionamento da figura original em relação ao centro de homotetia.

Cada etapa da atividade foi planejada para dois encontros de duas horas-aula. No primeiro encontro planejou-se a ambientação e manipulação dos applets dos pantógrafos virtuais. No segundo encontro, os alunos deveriam manipular os applets com maior atenção, observar os elementos da representação e responder as questões da folha de acompanhamento de atividade, conforme exposto no Quadro 1.

Quadro 1 - Organização do bloco de atividades do Pantógrafo

\begin{tabular}{|l|l|c|}
\hline \multicolumn{2}{|c|}{ Encontro 1 : Ambientação - 100 minutos (2h/a) } \\
\hline$N^{\circ}$ & \multicolumn{1}{|c|}{ Etapas } & Estimativa de Tempo \\
\hline 1 & Apresentação atividade e perguntas motivadoras & $20 \mathrm{~min}$ \\
\hline 2 & Localização e acesso ao site & $15 \mathrm{~min}$ \\
\hline 3 & Ambientação e experimentação do applet. & $40 \mathrm{~min}$ \\
\hline 4 & Registro do desenho feito no applet no Paint & $15 \mathrm{~min}$ \\
\hline 5 & Salvamento do arquivo & $10 \mathrm{~min}$ \\
\hline \multicolumn{2}{|c|}{ Encontro 2: Manipulação e observação -100 minutos $(2 \mathrm{~h} / \mathrm{a})$} \\
\hline 1 & Acesso ao site e a folha de atividade 8 & $10 \mathrm{~min}$ \\
\hline 2 & Manipulação dos applets - Pantógrafo virtual & $40 \mathrm{~min}$ \\
\hline 3 & Execução das respostas da folha de atividades 8 & $45 \mathrm{~min}$ \\
\hline 4 & Salvamento do arquivo & $5 \mathrm{~min}$ \\
\hline
\end{tabular}

No primeiro momento do primeiro encontro da atividade, perguntas sobre a função dos pantógrafos serviram para motivar cada dupla a observar e refletir sobre a movimentação dos applets. No segundo momento, a página do applet dos pantógrafos virtuais foi acessada por cada dupla de estudantes que fizeram as primeiras movimentações, alterando os valores a e $\underline{b}$ da aplicação (Figura 2) e observando o resultado dessas alterações no desenho.

O applet dos pantógrafos virtuais não disponibiliza a opção de salvamento dos arquivos; por isso, após a manipulação dos applets do pantógrafo virtual de Scheiner, cada dupla acionou a tecla print screen do computador para fotografar a tela dos monitores e respectivos desenhos. A imagem da tela gerada pelo print screen foi colada em um arquivo do Paint. Para fins de registro da atividade, o arquivo foi identificado com o nome da dupla de alunos e salvo em uma pasta na área de trabalho do computador. Esse recurso já havia sido utilizado com as turmas na atividade do Ctrl C, Ctrl V (IZAR e BAIRRAL, 2013) ${ }^{8}$, concebida para instigar a percepção dos participantes sobre como uma imagem pode ser ampliada ou reduzida proporcionalmente.

\footnotetext{
${ }^{8}$ Para ver um material curricular elaborado com esse tema acesse http://www.gepeticem.ufrrj.br/portal/materiaiscurriculares/ctrl-c-ctrl-v-no-paint-2/ e http://www.gepeticem.ufrrj.br/portal/videogallery/retangulos-mal-comportados/.
} 
No segundo encontro do bloco de atividades do pantógrafo, cada dupla deveria acessar o applet do site da Associazione Macchine Matematiche e responder questões relativas à atividade , disponibilizadas em um arquivo do Word na área de trabalho de cada computador. A manipulação e a interação com os pantógrafos virtuais motivou a observação das características das figuras desenhadas por cada pantógrafo (posição, tamanho dos lados, abertura de ângulos, etc.), além de suas características estruturais. Após a atividade, os arquivos também foram identificados e salvos na área de trabalho dos computadores. O Quadro 2 mostra as perguntas da Folha de Atividade.

\section{Quadro 2 - Folha de Atividades}

\begin{tabular}{|c|c|}
\hline 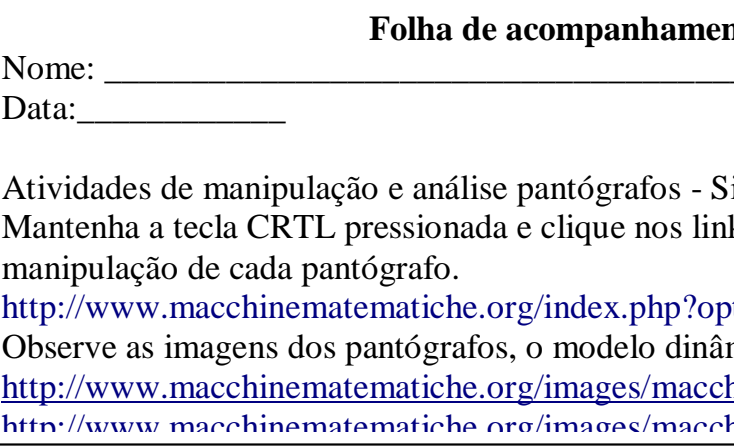 & $\begin{array}{l}\text { Associazione Macchine Matematique } \\
\text { dados a seguir para ter acesso aos Applets de } \\
\text { n=com_content\&view=article\&id=75\&Itemid=152 } \\
\text { co do site e responda as perguntas a seguir. } \\
\text { e/trasformazioni/simulazioni/traslatore.html } \\
\text { e/trasfrrmarinni/simularinni/nmntetia html }\end{array}$ \\
\hline $\begin{array}{l}\text { Parte 1 - Estrutura do pantógrafo. } \\
\text { 1.1 Quantas hastes compõem o pantógrafo? } \\
\text { Observe o tamanho de cada haste do pantógrafo } \\
\text { (AB,BC,CD,AD). São congruentes? Quais são as } \\
\text { que possuem o mesmo tamanho? } \\
\text { 1.2 Que figura geométrica é formada pelas hastes } \\
\text { ABCD e BCQP? } \\
\text { No pantógrafo, há alguns vértices fixos (no plano } \\
\text { ou forçados a se mover de uma determinada } \\
\text { maneira - vértices limitados) e outros livres para se } \\
\text { mover no plano (vértices livres). } \\
\text { 1.3 Quais são os vértices livres? } \\
1.4 \text { Quais são os vértices limitados? } \\
1.5 \text { Por que são limitados? } \\
\text { Parte } 3 \text { - Como o pantógrafo trabalha? } \\
\text { 3.1 Que características do pantógrafo permitem a } \\
\text { manutenção da relação entre os pontos } \mathrm{P} \text { e } \mathrm{Q} \text { ? } \\
\text { 3.2 Justifique a resposta. }\end{array}$ & 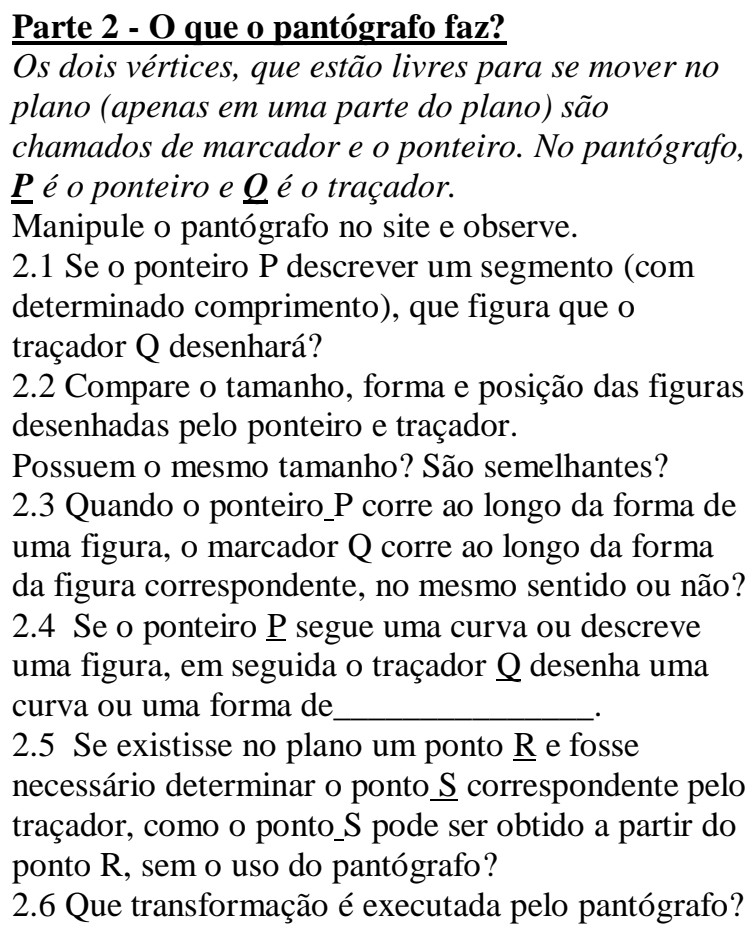 \\
\hline
\end{tabular}

Fonte: Elaborado pela autora, inspirada no Kit "TRANSFORMAZIONI".

As perguntas relativas à estrutura do pantógrafo de homotetia possuem a intenção de levar o aluno a identificar os polígonos formados pelas hastes e verificar a relação desses polígonos com a figura desenhada pelo instrumento. No caso do pantógrafo de Scheiner, observar os triângulos formados pelo pontos OAQ e OBP, o paralelismo entre os segmentos AQ e BP e entre OB e CQ, assim como o paralelogramo definido por ABCQ, conforme mostra a figura 4. A identificação do 
ponto fixo do sistema (O), dos pontos articulados (A, B e C) e dos pontos livres (P e Q) são importantes para verificar a relação entre a figura original e a figura transformada.

Figura 4 - Polígonos formados pelas hastes do pantógrafo de Scheiner.

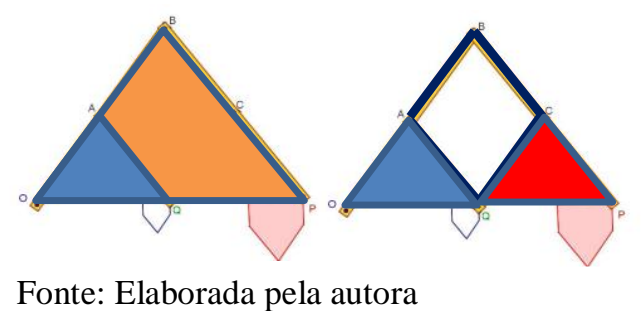

Fonte: Elaborada pela autora

\section{Considerações finais}

As atividades foram planejadas para os estudantes nativos digitais (PRENSKLY, 2001) que nasceram convivendo com os recursos da tecnologia digital e, por esse motivo, a maioria dos aprendizes gostou da forma diferenciada de desenvolvimento do conteúdo. Os alunos mostraram que aprenderam o conceito de homotetia, assim como as características da transformação (observação da semelhança, o comportamento dos pantógrafos e a posição das figuras) envolvidas no bloco de atividades dos pantógrafos.

Essa proposta convida o docente a uma reflexão sobre a necessidade de elaboração de atividades que contemplem as habilidades dos alunos nativos digitais, assim como as possibilidades de implementação de atividades que associem tais habilidades às atividades e conteúdos desenvolvidos no cotidiano escolar. Também é necessário destacar a importância da atualização do corpo docente para a utilização das TIC, particularmente, como ilustra aqui, de recursos dinâmicos disponibilidades na Internet ou em sítios institucionais.

\section{Referências}

BAIRRAL, M. A. Discurso, Interação e Aprendizagem Matemática em Ambientes Virtuais a Distância. Rio de Janeiro: Edur, 2007.

BAIRRAL. M. A. Tecnologias da Informação e Comunicação na Formação e Educação Matemática. Vol. I. Rio de Janeiro: Edur, 2009.

FERREIRA, A. B. H.. Dicionário Aurélio Básico da Língua Portuguesa. Rio de Janeiro: Nova Fronteira, 1986.

IZAR, S. B., BAIRRAL, M. A. Aplicativos dinâmicos e cultura visual na exploração do conceito de homotetia. Revista Brasileira de Expressão Gráfica (RBEG), v. 4, n. 1, p. 1-19, 2016.

IZAR, S. B.; BAIRRAL, M. A. Ctrl C, Crtl V: alunos ampliando e reduzindo figuras no Paintbrush. Anais ... CONGRESSO IBERO-AMERICANO DE EDUCAÇÃO MATEMÁTICA, 7., 2013, Montevideo. Anais... Montevideo: Colégio Seminário, 2013. p. 6285-6292. 
OLIVEIRA, M. K. de Vygotsky - Coleção Grandes Educadores. [Filme-vídeo]. Produção Régis Horta, direção de Régis Horta. São Paulo, Atta Midia e Educação, 2010. DVD, 45min. Color, som.

PRENSKY, M. Nativos e Imigrantes Digitais. MCB University Press, Vol. 9 No. 5, October 2001. Disponível em: <http://www.marcprensky.com/writing/Prensky\%20-

20Digital\%20Natives,\%20Digital\%20Immigrants\%20-\%20Part1.pdf> Acesso: 03 dez. 2016.

RODRIGUES, M. H. W. L. Transformações Pontuais: Fundamentos, anotações e exercícios. Rio de Janeiro: (Apostila da disciplina Teoria do Desenho Geométrico II, oferecida na Escola de Belas Artes da Universidade Federal do Rio de Janeiro), 1997.

VELOSO, E. Geometria, temas atuais: materiais para professores. Instituto de Inovação Educacional. Lisboa. 1998.

Submetido em novembro de 2016 Aprovado em dezembro de 2016 